\title{
Usage of Interactive Event Timelines in Collaborative Digital Tabletops with Automation
}

\author{
Y.-L. Betty Chang, Stacey D. Scott, and Mark Hancock ${ }^{1}$
}

\begin{abstract}
Tabletop computers are increasingly being used for complex scenarios, such as emergency response. In such scenarios, maintaining situation awareness of dynamic changes automated by the system is crucial for users to make optimal decisions. If the system does not provide collaborators with appropriate feedback, they can become confused and "out-of-the-loop" about the current system state, leading to non-optimal decisions or actions. To enhance situation awareness of dynamic changes occurring in the collaborative tabletop environment, we designed an interactive event timeline to enable exploration of historical system events. We conducted a user study to understand how various design alternatives of interactive event timelines impacted situation awareness in the context of a cooperative tabletop game. Our initial results showed that, on average, all groups scored high on their combined level of situation awareness, regardless of the given timeline designs. To better understand what role the timelines played for the groups, we conducted an in-depth video analysis. Participants used the timelines mostly for perceiving new changes by interacting with the detailed information. The high-level information was beneficial for projecting future system states. The information presented in the timeline was considered as the correct historical account and was used for negotiating participants' knowledge of the changes. We al-
\end{abstract}

\footnotetext{
This is the author's version of this book chapter. It is posted here for your personal use. Not for redistribution. The definitive version of record should be cited as:

Chang YL.B., Scott S.D., Hancock M. (2016) Usage of Interactive Event Timelines in Collaborative Digital Tabletops Involving Automation. In: Anslow C., Campos P., Jorge J. (eds) Collaboration Meets Interactive Spaces. Springer, Cham. https://doi.org/10.1007/978-3-319-45853$\underline{3} 10$

${ }^{1}$ Y.-L. Betty Chang

Games Institute / Systems Design Engineering, University of Waterloo, Waterloo, ON, Canada; e-mail: betty.chang@uwaterloo.ca

Stacey D. Scott

Games Institute / Systems Design Engineering, University of Waterloo, Waterloo, ON, Canada; e-mail: stacey.scott@uwaterloo.ca

Mark Hancock

Games Institute / Systems Design Engineering, University of Waterloo, Waterloo, ON, Canada; e-mail:mark.hancock@uwaterloo.ca
} 
so report on how other system components, in addition to the interactive timelines, were used for situation awareness maintenance. Finally, we discuss implications for designing interactive event timelines for co-located collaborative systems involving automated events.

\section{Introduction}

There is a growing interest in using digital tabletops for co-located group activities that involve complex, often dynamically changing data. Given their ability to provide digital functionality for collaborative work while allowing for face-to-face communication, tabletop interfaces have been proposed for many domains, such as crisis and disaster management [6] and commercial maritime operations [5]. In such domains, decision-makers' awareness of the system state is crucial to the decision making quality [11]. However, when the system automates changes in system states but does not provide appropriate feedback via the user interface, human operators are left "out-of-the-loop" [22]; that is, they are unable to keep up with system changes. They may result in confusions and unable to make optimal decisions and interact when needed. As digital tabletop applications become more sophisticated and begin to incorporate more automation to manage the type of complex data inherent to many real-world application domains, keeping users in-theloop becomes an essential design requirement.

Due to a variety of potential distractors, tabletop applications cannot assume that users will attend to and notice all system changes. For example, conversing with collaborators at or near the tabletop, or attending to other devices being used in conjunction with the tabletop (e.g., a smart phone or tablet) can distract users. Moreover, a user may be called away from the tabletop temporarily. Consequently, a change occurring on the tabletop (automated, or made by another user) can be easily missed. However, existing tabletop application that incorporate dynamic provide little to no provision for situation awareness maintenance, such as displaying historical system data, and instead focus on novel interfaces for sharing or collaborating with the current, real-time view of the system state $[1,4]$.

As interactive event logs and timelines have been previously shown to reduce response time and improve decision accuracy for single-user applications involving automated system changes [21,35], we were interested in adapting such timelines to a co-located collaborative context on tabletop systems. We investigated two design factors: control placement (how many timelines for a group of users and where it is) and feedback location (where to display feedback upon interacting with the timeline). We seek to understand how these two design factors impact collaborative work and situation awareness of dynamic changes in collaborative tabletop applications. 
We evaluated the design factors in the context of a popular three- to fourplayer collaborative tabletop board game, Pandemic ${ }^{2}$, for several reasons. This game requires intense strategy discussions, resource management, and advance planning to prevent the world from epidemic outbreaks. Moreover, Wallace et al. [47] found that their digital tabletop version of the Pandemic game elicited the aforementioned out-of-the-loop automation problem, due to the amount and complexity of changes as well as the fact that players were not constantly paying attention to the tabletop interface.

Our study involved two phases. Phase 1 involved a controlled experimental design that tested the two design factors by asking participants to play three short partial games in which they used three different timeline alternatives. In Phase 2, participants completed a full game from start to finish using a configurable version of the timeline that allowed them to utilize any combination of the control placement and feedback location at any time.

The results from a detailed analysis of Phase 1, previously published in Chang et al. [3], revealed that replicated timelines — where each player had their own copy in their personal space - encouraged more timeline interactions. More interactions with the timeline correlated with higher levels of individual situation awareness. Despite the differences in individual situation awareness, overall, groups were found to have high combined levels of situation awareness in all conditions. In this article, we expand on this prior work by presenting the results of an in-depth video analysis of players' situation awareness maintenance behaviour in Phase 2, which provide a better understanding of how system features, including the timelines, were used to understand dynamic changes driven by the system. The analysis revealed that the timelines were useful as both static and interactive visualizations, and they were mostly used to investigate recent dynamic changes by the system. Timelines were used only occasionally to strategize and prioritize tasks while another system feature, discard pile, was used primarily for this purpose.

We first present the related work on situation awareness and workspace awareness to contextualize our research within the literatures on awareness of dynamic changes in a group context. Next, we present the conceptual design of our interactive event timelines and the timeline design alternatives motivated by the previous work on awareness support in tabletop research. We then introduce the Pandemic game case study and describe our timeline designs. We then present the study method and results, focusing on the video analysis results of timeline usages based on Phase 2 of the study. Finally, we discuss the implications of our findings on timeline designs and conclude with future work.

${ }^{2}$ The Pandemic game was published by Z-Man Games, used with permission. 


\section{Related Work}

This work investigated how players maintain awareness of the changing system state in a collaborative, digital tabletop, board game. There has been substantial research on the concept of awareness (and its many forms) in the Human Factors and Human-Computer Interaction literature e.g., $[31,36]$. In this section, we provide an overview of relevant research specifically in the areas of situation awareness, team situation awareness, and workspace awareness. We discuss related work in digital tabletops in section 3 below to motivate the design factors studied.

\subsection{Situation Awareness (SA)}

Situation awareness (SA) describes a person's awareness of the environment, and has been applied to many domains, including military combat [10], aviation [41], and nuclear plant operation [2]. Endsley [11] defined situation awareness (SA) as the perception of changes in the system state (level 1), the comprehension of the changes (level 2), and the projection of future system states (level 3 ). The second level of situation awareness requires people to connect multiple pieces of knowledge (level 1) to infer their meaning and form an understanding of the perceived changes. The third level describes the ability to predict future states of the system based on the person's understanding (level 2).

The automation literature has observed that the phenomenon of change blindness is a key cause of deficient situation awareness in automated systems [7]. Change blindness refers to a person's inability to recognize changes in the environment after interruption or deviation in attention [30]. The interruption recovery literature has explored the use of persistent, interactive information displays to mitigate change blindness and to rapidly improve situation awareness following an interruption to the task in systems with dynamically changing data $[35,38,40]$.

Sasangohar et al. [35] studied interactive event timelines that allowed users to highlight historical events on a main task display (a map) located on a large wall display by interacting with event bookmarks, which were displayed on a graphical timeline located on a secondary handheld display. Their results showed that the timelines allowed people to quickly gain awareness of missed events and helped reduce recovery time and improve decision accuracy after interruptions. They argued that the interactive event timelines provided a "simplified representation of important events [that] facilitated the quick encoding of perceptual information and minimized the visual search" [35:1155]. On a large digital tabletop interface, promoting situation awareness while minimizing visual search across the entire interface is an important design goal. Thus, our work applies this interactive event timeline concept to digital tabletops involving automation.

Previous research has largely focused on the design of awareness displays to support situation awareness for individuals [21,35,38]; our research expands on this by applying interactive event timeline to multi-user tabletop environments. 


\subsection{Team Situation Awareness (TSA)}

As we aim to support team environments where users have a shared goal, examining individual SA of the system may not be sufficient to understand the collaborative process of gathering situation awareness and strategizing as a group.

Team situation awareness (TSA) is the team members' overlapping knowledge of the situation as well as the full situation awareness required for individuals to successfully coordinate actions and complete the shared goal [34]. Previous work generally agrees that TSA requires high levels of individual SA and communication among team members $[8,13,48]$. Thus, much of the research in TSA has focused on individual tool design (i.e. to facilitate individual SA, discussed in the previous section) and analysis of communication and coordination behaviours to provide design implications and create advance measurements $[8,13,28,34]$.

Theoretical models of TSA have also been developed [8,33,34]. However, they tend to focus on high level processes [8,34], such as shared goals, communication, team members' background, and teamwork. In this project, we examine how specific features of our system were leveraged by our participants to maintain and communicate situation awareness.

\subsection{Workspace Awareness}

Extensive research has shown the value and the information richness provided by the objects, people, and environment in co-located collaborative settings $[16,19,29]$. While situation awareness focuses on a person's knowledge of a system's state, workspace awareness describes a person's knowledge of their collaborators and their actions within a shared (physical or virtual) workspace [29].

In distributed settings, workspace awareness has been supported through techniques such as virtual embodiment (e.g., telepointers [16], virtual arms [45], avatars [24]). Although a significant amount of workspace awareness information can be gained "for free" in a co-located tabletop setting [16], the distance between collaborators and the complexity of some tabletop interfaces can hinders people's ability to observe all activities occurring in the shared workspace, especially for interactions happening in collaborators' personal spaces [37]. In this project, we seek to preserve users' workspace awareness while providing them with interactive event timelines to maintain situation awareness. Thus, we tested two factors relevant to the design of timelines for supporting workspace awareness in the context of tabletop systems, and we discuss these factors in section 3.2 below. 


\section{Awareness Support for Tabletop Systems involving Automation}

Traditionally, automation was used to reduce manual workload or change the states of physical materials by using mechanical machines [27]. Now, automation is also used to reduce mental workload, and may involve changing the state of virtual objects, such as automatically updating data visualizations based on underlying sources. However, these automated changes can negatively impact situation awareness, often due to change blindness or state changes not being displayed. As illustrated in Fig. 1A, people at a digital tabletop can be unaware of a change occurring in the system interface due to the large size of the display or other competing demands for their attention, such as conversing with a teammate. Moreover, even when a change occurs within a person's field of view, they may still miss the change due to limited attentional capacity.

\subsection{Situation Awareness Support for Tabletop Systems}

Substantial tabletop research has investigated interaction techniques for digital object manipulation, menu invocation, information sharing, and tangible interaction $[23,42,46,49]$. Significant work has also been done on information visualization, coordination and collaboration styles, and control widgets for collaborative tabletop systems $[20,25,43]$. As more sophisticated tabletop applications are developed to support complex task domains $[1,5,6]$, application tools that allow maintenance of awareness of dynamic changes will become essential. To date, no such tools have been tested in collaborative tabletop environments, and existing tabletop applications involving dynamic data focus on supporting current, real-time view of the system state in collaborative work [1,4]. This work is a first step towards addressing this gap. 


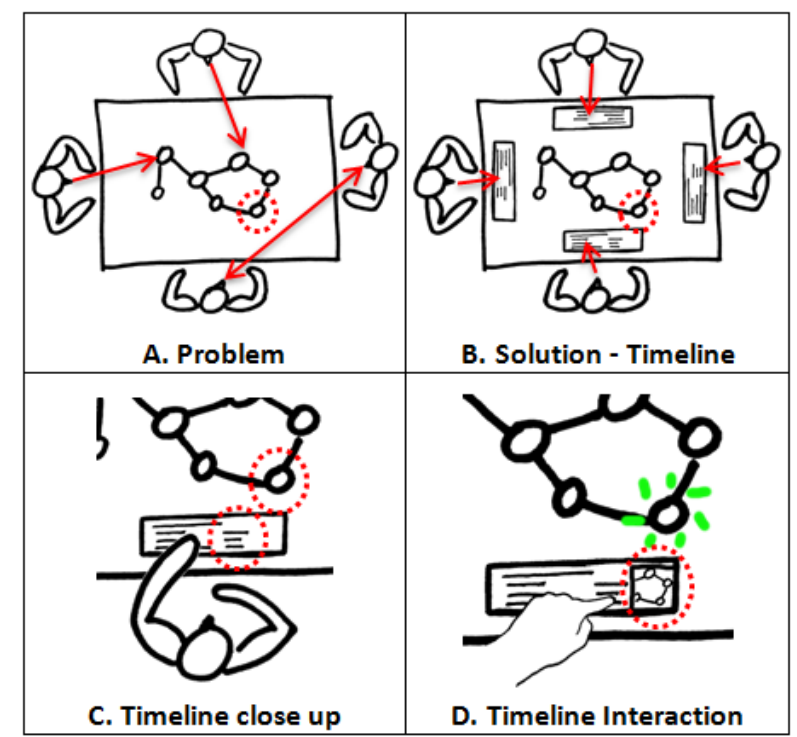

Fig. 1 Conceptual design of the interactive event timelines. A) Problem: users can miss automated changes if they are engaging in conversations or focusing on another part of the tabletop display (red arrows show attentional focus). B) Solution: timelines provide a way for users to view and explore changes. C) New changes are appended to the timeline, and D) users can interact with the timelines to locate the changes on the shared area (highlighted node) and on the timeline (graph cutout on the right of the timeline).

\subsection{Conceptual Design}

To address these issues introduced by the use of automation in a digital tabletop system, we explore using interactive event timelines to provide persistent information of historical system events. Moreover, such timelines provide the information in a visual form that can fit within a person's field of view, despite the large size of the table. To gain awareness of the current system state, a person can examine and explore the timeline, which provides an overview of the historical events (Fig. 1B and Fig. 1C). To get more in-depth information, they can invoke further feedback on the shared display or on their personal areas (Fig. 1D). Based on the existing literature, we considered two key design factors in the design of these timelines: control placement and feedback location.

\subsubsection{Control Placement}


The event timeline is a visualization of historical events as well as a control for invoking detailed information of the automated changes. It was unclear to how to best distribute and place the timelines to best support workspace awareness and situation awareness in a group setting.

Morris et al. [25] compared providing individual replicated system controls around the border of a tabletop system with a single, shared control in the centre for a collaborative photo tagging application. They found that while individual controls were preferred, the groups were more collaborative (i.e., more labels per image) when using the shared controls. This result suggests that a shared timeline may contribute to more collaborative work and improved team situation awareness (e.g., joint investigation for all team members). However, it is unclear to how well shared timelines support individual situation awareness since users need to coordinate their use of the timelines.

Ha et al. [17] compared direct touch and mouse pointers for a two-player competitive image search game on digital tabletops, and their results show that the direct touch condition allowed for higher levels of workspace awareness and resulted in quicker response to opponents' moves. Nacenta et al. [26] studied five different interaction techniques for selecting, moving, and rotating images for two collaborative tasks: an image sorting game and a storyboarding activity. They similarly found that the interaction technique requiring explicit input in the shared space (i.e., drag-and-drop) allowed for easier tracking of collaborators' actions and helped avoid conflicting actions. While participants may have higher workspace awareness using the shared control, it was unclear how individual versus shared timelines would impact participants' situation awareness. Providing replicated timelines would ensure each person could view and manipulate the timeline for the purpose of maintaining situation awareness. As the current research still lacks understanding in how the placement of timelines would impact users' situation awareness, we examine the control placement factor.

\subsubsection{Feedback Location}

Another design consideration is where to provide the visual feedback related to historic system events being explored by a user. Information about the event can be displayed locally (on the timeline) or on the shared area of the tabletop. These design alternatives may better facilitate either individual control or group function, respectively [15]. Displaying feedback on the timeline provides a consistent location to look for the information, and it fits into a person's field of view. On the other hand, feedback in the shared area provides more contextual information of the overall situation to the individual. This feedback location also better facilitates feedthrough - the observation of shared artifacts in the workspace to gain awareness of collaborators' actions and work progress [29] - by making collaborators' actions more visible to the whole team. However, the size of the display may still necessitate searching for the feedback in the shared workspace, making situation 
awareness maintenance more difficult for individuals. Moreover, other users' feedback on the shared area may make searching more difficult and distract users.

Existing work that explored the impact of specific input methods and interaction techniques on workspace awareness $[17,26]$ provides insights that helped us hypothesize how the different feedback locations may impact workspace awareness. However, our timelines were designed for situation awareness maintenance, which is a different goal from the previous work. Thus, the timeline's impact on situation awareness and the trade-off between providing awareness and reducing distraction needed further investigation.

\section{Studying Interactive Event Timelines in the Pandemic Game}

Our literature review revealed a gap in supporting situation awareness in complex tabletop applications involving dynamic automated changes. While interactive event timelines were shown to improve interruption recovery time and decision accuracy, there still lacks understanding in how to adapt timelines to a tabletop application for situation awareness support. Thus, we designed an interactive event timeline and chose a cooperative board game as our study context. We describe the study context and timeline design in this section, and we present the study design in the section 5 below.

The cooperative board game, Pandemic, was selected for several reasons. Games allow for a more rapid, human-centred prototyping process, since it is easy to recruit "subject matter experts" of popular games. We can more control in manipulating parameters, such as degree of difficulty. Moreover, the digital tabletop version of the Pandemic game by Wallace et al. [47] was shown to elicit situation awareness deficiencies due to automation.

Pandemic is a commercial board game for three-four players, requiring intense collaborative activities, such as forecasting game states, advance planning, and managing resources. Players work together as a team, with distinct roles and abilities, to save the world from epidemic outbreaks. Players win by curing all the diseases, and lose if they run out of time (not having enough cards to draw from) or if the game state is out of control (too many outbreaks or diseases). During each turn, a player completes four actions through careful planning and strategizing. Then, they draw player cards, which are collected to trade for the cure (i.e., to win the game). At the end of a player's turn, the team acts as the game board (opponent) and draws infection cards that determine which cities are infected with new diseases (in the original board game, players place wooden cubes (diseases) onto the game map based on the cards drawn). Outbreaks and epidemics are critical events that increase the difficulty of the game, and players have to stay aware of them to effectively strategize. 


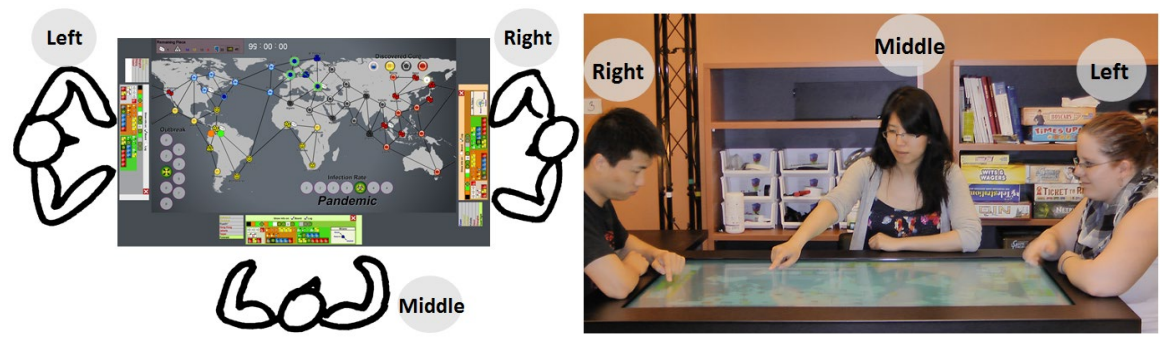

Fig. 2 The Pandemic board game was used to study situation awareness support in tabletop systems. (Left) a screenshot of the game interface, labeled with participant seating locations, based on the orientation of the game map. (Right) A group was playing the game.

Our digital tabletop adaptation version of Pandemic (Fig. 2) provided automation to help reduce manual workload and to enforce rules. For example, the system automated game board (the opponent) actions by placing disease cubes based on cards drawn, or outbreak and epidemic events. As participants may be confused by the complexity and number of changes that can happen all at once or in quick successions, the game conveyed the changes through the following three system features.

Board. The changes were reflected on the game board, including displaying disease cubes on the map and counters around the map (e.g., cards left, epidemic counters, and cubes left). Moreover, after automated system events, three seconds of system animations appeared to highlight the changes on the relevant cities (e.g., Fig. 3L). Different types of system animation were used to represent infection, outbreak, and epidemic events.

Infection Discard Pile. The system provided a limited history of previous infected cities in a textual log format, contained in the infection discard pile (see Fig. 3M). The pile was periodically emptied into the infection draw pile when an epidemic event occurred so it only contained limited history since the last epidemic. Players could open the discard pile via a button on the top left of the interface. It initially opened at the centre of the game map, and can be moved by dragging the pile.

Interactive Event Timeline. The interactive event timelines provided a complete record of events that happened throughout the game, and is presented in section 4.1 . 


\subsection{Interactive Event Timeline Design}

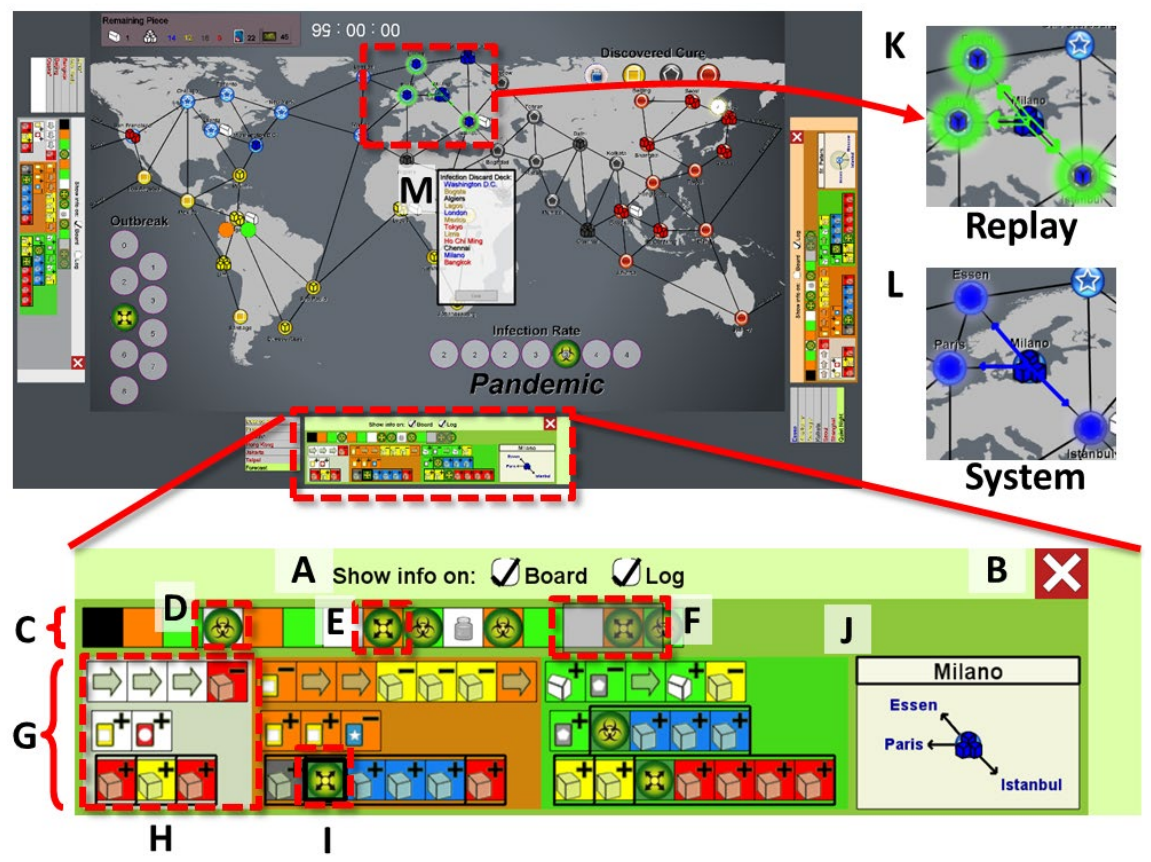

Fig. 3 Design of the interactive event timeline (configurable version). Users could (A) toggle the feedback location on the board and on the timeline as well as (B) close and open it at any time. (C) The overview bar showed all players' turns so far with symbols denoting important game events, such as (D) epidemics and (E) outbreaks. $(G)$ A viewport was used for selecting a timeframe to show in the detail view. (H) A player's turn contained three rows, corresponding to the three game phases. Each block represented an action carried out by either the player or the system, and black bounding boxes grouped related game events. (I) Selected event had a thick black bounding box. Location details of the selected event was (J) shown on the timeline as a map cut-out and (K) highlighted through a replay animation on the map (in contrast to the (L) system animation). (M) The infection discard pile is shown at the centre of the map by default. For full details, see Chang et al. [3].

We designed an interactive event timeline (Fig. 3) to improve players' awareness of the game's automated actions and of other players' actions. The design was based on a task analysis of experienced to expert players playing the Pandemic game. The timeline allowed players to explore prior game events, including both player and automated actions. The timeline was designed to fit into a player's personal territory on the tabletop, based on prior research on tabletop territoriality [37]. Moreover, it persisted on the game board, allowing players to interact with it 
at any time. When new changes happened, they were appended to all timelines in the game, and they automatically scrolled to show the current game turn.

The timeline consisted of two main components: an overview (Fig. 3C) and a detail view (Fig. 3G). The overview showed an overview of all turns so far in chronological order, colour-coded by the in-game player colour (orange, green, and white). Critical events were marked with symbols on turns in which they occurred. To navigate through the game history, players could drag the viewport (Fig. 3F) or tap on a given turn to reveal details of the turns of interest in realtime.

The detail view (Fig. 3G) contained the currently selected player turns. Each turn consisted of three rows corresponding to the three phases in the game (Fig. $3 \mathrm{H})$ : 1) player actions, 2) cards drawn for players by the system, and 3) cities infected by the system. Each block represented one game event with a symbol denoting the type of event and colour derived from the colour coding scheme in the Pandemic board game. Selecting a game event displayed a replay animation on the shared game map (if feedback on the board was enabled) and/or detailed information next to the timeline (if feedback on the timeline was enabled) (Fig. 3J-Fig. $3 \mathrm{~K})$.

\section{Study}

We conducted a laboratory-based study to understand how the two design factors, feedback location and control placement, impact users' situation awareness and timeline usage. Participants played the Pandemic game with different design alternatives of the interactive event timeline, and answered questionnaires for us to evaluate their situation awareness and experience.

\subsection{Participants}

Participants were recruited from the local community, specifically targeting experienced Pandemic players. Players had to sign up in groups of three. Thirty-six paid participants (twenty-three male, thirteen female, ages twenty-two to thirtysix) were recruited, with all team members having previous experiences playing Pandemic prior to the study. For this book chapter, the participants are denoted as $\mathrm{P}_{\text {group number, seating position. }}$ For example, $\mathrm{P}_{1 \text {, right }}$ denotes the right player in Group 1 (based on the orientation of the game map). 


\subsection{Equipment \& Setting}

Each group of participants was seated in the lab around a $148 \times 95 \mathrm{~cm}$ digital table $(3840 \times 2160$ pixel, $121 \times 67 \mathrm{~cm}$ for screen size $)$ with an embedded PQ Labs frame to detect touch input. Two participants sat at the short edge, and one participant at the long edge, to avoid the situation of one participant seeing the game board upside down (see Fig. 2). The computer was running 64-bit Windows 7 using an Intel® Xeon® CPU E5-1603@2.80 GHz with 4 GB of RAM. Two digital camcorders were placed at different angles to capture the game sessions.

\subsection{Study Design}

There were two study phases. Phase 1 (Pandemic Challenges) was conducted using a mixed design, testing two factors:

- Control placement (between-subjects): 2 levels (shared, individual)

- Feedback location (within-subjects): 3 levels (next-to-timeline, on-board, both)

For control placement, half of the groups used the shared controls and the other half used the replicated individual controls. The order of the three feedback locations was counterbalanced. In this phase, we seek to understand these two factors' impacts on participants' situation awareness.

In Phase 2 (Full Game), participants played a full game with a configurable version of the timeline, in which a group could open up to three timelines that could be moved anywhere on the tabletop. They could minimize and reopen these timelines at any time (Fig. 3B). Each timeline allowed players to indicate where the feedback generated by that specific timeline was displayed (next-to-timeline, on-board, or both) via toggle widgets at the top of the timeline (Fig. 3A). All groups had the same game setup. We seek to observe a more realistic usage of the timeline to inform further improvements and to understand how it was used to facilitate the situation awareness maintenance.

\subsection{Procedure}

The study sessions lasted approximately two and half to three hours. Participants first completed consent forms and background questionnaires, and then completed the two study phases: 1) Pandemic challenges and 2) a play through of a full game. 


\subsubsection{Phase 1 - Pandemic Challenges}

After the researcher explained the game interface, participants played with the Pandemic game without using timelines for ten minutes and completed the gameplay questionnaire (study questionnaires are discussed in section 5.5 below). Then, with the same procedure, participants practiced on the same timeline variant they would see in the first Pandemic challenge.

For each trial, participants started in the middle of an ongoing Pandemic game and played for two rounds (two turns for each player). We constructed three initial game states (scenarios) from real gameplay with some controlled parameters, such as the number of critical events that happened and the number of cures discovered. The order of the initial game states was randomly selected.

Players individually completed post-condition questionnaires, which consisted of both the gameplay and the situation awareness (SA) questionnaires. The order of the three SA questionnaires was randomly selected. Participants were asked to rank their preferences of the timeline alternatives at the end of this phase and to provide free-form feedback.

\subsubsection{Phase 2 - Full Game}

After the researcher explained the configurable timeline, participants played a full game. Then, they completed the gameplay questionnaire with a free form area for any additional comments.

Finally, the researcher debriefed the participants with the goal and details of the study, and conducted an unstructured interview to receive any additional feedback.

\subsection{Data Collection}

During gameplay, we collected various data, including video recordings from two different angles, screen recordings, computer logs, audio recordings, and questionnaires. The computer logs captured all touch interactions on the timeline, e.g., tap, rotate, open, and close timelines as well as toggle feedback locations.

Two questionnaires were used to evaluate participants' gameplay experience and situation awareness. The gameplay questionnaire consisted of the Player Experience of Need Satisfaction (PENS) survey [32] and the NASA Task Load Index (NASA-TLX) survey [18]. We developed the situation awareness questionnaire by following the steps outlined in the SAGAT methodology $[9,12]$. More details on the questionnaire can be found in Chang et al. [3]. 


\subsection{Quantitative Analysis}

Each individual's situation awareness questionnaire results (from Phase 1) were scored as correct (1), partially-correct (0.5), and incorrect (0) for each question. We analyzed the situation awareness questionnaires using a 2 (control placement) $\times 3$ (feedback location) repeated measures analysis of variance (RM-ANOVA). See full details of the quantitative analysis in Chang et al. [3].

\subsection{Qualitative Analysis}

For Phase 2 (full game), two researchers analyzed eight full game sessions with an open video coding process. One researcher watched the videos and took notes of participants' discussions and activities related to timeline usages and situation awareness maintenance. An initial set of codes was then established, and two researchers coded for players' interactions with the components in the game and their discussion with teammates, e.g., interaction with timeline and discard pile, pointing at the game board, and deictic references to game cities as well as announcement, narration and discussion of automated events. The codes were revised until an acceptable inter-rater reliability was reached $(79.39 \%)$, and then the rest of the videos were coded.

Next, we focused on codes most relevant to participants' situation awareness maintenance, including 1) looked at or touched the timelines, 2) opened and closed the timelines, 3) toggled feedback locations, 4) opened the infection discard pile, 5) discussed automated game events, and 6) corrected each other's knowledge of the automated events. For all instances, we classified the purpose behind the observed actions and discussions as follows:

- Investigation of automation results: the coded instance was conducted for the purpose of finding out the type and location of an automated event that took place as well as connections between automated events.

- Prioritization: the coded instance was conducted for the purpose of gathering information to predict future game state and prioritize player actions.

- Other: the coded instance was for any other purposes, such as played with the timelines, rotated the timelines, and toggled feedback locations at the start of the game for learning. This category also included instances that could not be classified by the researchers (i.e., insufficient information).

The instances were also classified based on whether the participants achieved their goals, such as correct (successfully obtained correct information), incorrect (successfully obtained, but information incorrect), incomplete (failed to obtain information), and unknown (researchers were unable to classify its successfulness). 
Next, to understand how players made use of various system features for situation awareness maintenance, we sequenced the codes based on game events investigated by participants. We also extracted the treat diseases game actions from the software $\log$ and included them in the sequence to understand the impact of various system features' usages on decision making. We classified each sequence based on its purpose and whether it led to a success in achieving the goal.

Through the video analysis, it became apparent to us that the codes classified under automation results were most closely related to the perception and comprehension levels of situation awareness. In the process of gathering results of automation, participants were investigating and verifying the exact new automated events that took place to understand the overall game state. On the other hand, the prioritization actions were most relevant to the projection of future game states as participants gather information to determine their urgency.

\section{$6 \quad$ Results}

In this section, we first summarize the quantitative results from Phase 1 on the control placement and feedback location factors (details can be found in our previous publication [3]) to motivate our video analysis. Next, we present our video analysis results on timeline configurations and describe how timelines and other system features were used for the three levels of situation awareness.

\subsection{Summary of Phase 1 Findings}

The analysis of Phase 1 data revealed that participants who used individual timeline controls had higher situation awareness scores than participants who shared a timeline within the group $\left(\mathrm{M}_{\text {individual }}=0.71, \mathrm{M}_{\text {shared }}=0.67, F_{1,28}=4.7, p=.04\right)$. Participants using individual timeline also interacted with their timelines more on average in each condition $\left(\mathrm{M}_{\text {individual }}=12.5, \mathrm{M}_{\text {shared }}=5.6, F_{1,10}=6.2, p=.03\right)$, including navigating and tapping on game events. This finding showed the benefits of the timelines for improved situation awareness, and a partial correlation (control for group) confirmed that there was a positive correlation between interaction with timelines and situation awareness scores $\left(r_{105}=.20, p=.04\right)$.

Although no main effects were found across feedback locations, feedback both on the game board and on timeline was most preferred as it provided the best of both worlds. For feedback on timeline, it allowed for quick navigation of game events while participants can fixate on the same area. However, participants reported that timelines felt disconnected from the game. Feedback on the board provided contextual information on the map, but participants also reported distrac- 
tions and confusion associated with board feedback; we will discuss this problem further in section 6.4 below.

Next, we investigated the groups' interactions with the timelines. We calculated a group situation awareness score by taking the best situation awareness score achieved by any one member for each question and then taking the average of these best scores. Overall, groups had high aggregated group situation awareness scores $(M=.87, S D=.06)$. However, there was no main effects for control placement $\left(F_{1,10}<0.1, p=.94\right)$ nor for feedback location $\left(F_{2,20}=1.2, p=.33\right)$. In the shared condition, the participant who interacted with the timeline most frequently was defined as the primary user, and the rest were secondary users. However, there was no difference in the individual situation awareness score between the primary and secondary users.

We hypothesized that the information participants gathered from the timelines was shared with the group; thus, the secondary users benefited from the primary users' interactions. Moreover, participants might have gathered situation awareness information through other components in the tabletop interface. Thus, we decided to follow-up with video analysis on the Phase 2 data to better understand how participants used various system features, including the timelines, to gain situation awareness.

\subsection{Timeline Configurations}

Our data analysis revealed that participants made use of the timelines in Phase 2, and kept it open for the most of the time. We then investigated the percent of time each feedback mode were kept on. When participants first started the gameplay, the timelines were set to show no feedback and were closed. However, if the game crashed and restarted (happened to two groups), the timelines were opened with both feedback locations on by default. The time during game crash was excluded from the analysis. For one group, the participants' timeline configurations before and after the game crash were different, and they did not all reconfigure their timelines. Thus, for this group, the time after the game crash was excluded.

As depicted in Fig. 4 Left, Both feedback was the most popular mode $(M=$ $60.82 \%, S D=42.02 \%)$, followed by Timeline Only $(M=30.05 \%, S D=40.32 \%)$ and Closed $(M=6.38 \%, S D=17.93 \%)$. Board Only feedback $(M=1.37 \%, S D=$ $7.31 \%)$ and None (while the timeline was open) $(M=1.37 \%, S D=1.59 \%)$ were the least kept mode. This distribution was consistent with participants' feedback and our observations, since the Both configuration was also rated as most preferred in Phase 1. Some participants reported noticing interference between their own feedback and others' feedback on the board, which was likely why the second-most frequent configuration was Timeline Only. While one player used Board Only more extensively ( $\mathrm{P}_{4, \text { right: }} 14.35$ minutes $)$, the rest of the participants almost never kept their timelines in this mode ( $M=2.6$ seconds, $S D=3.69$ seconds $)$. This 
was likely due to the need to search for the replay animation on the map as well as the interference problem. Although the percent of time in the None configuration might be a result of intermediate time between toggling feedback locations, the video analysis presented in section 6.4 below showed some benefits of the timeline as a static visualization.

Participants occasionally switched to different timeline alternatives throughout the game, but it was difficult to determine their intention based on the observable actions as there was no verbal explanation in most cases (only 6/31 cases could be clearly identified as related to understanding automated events).
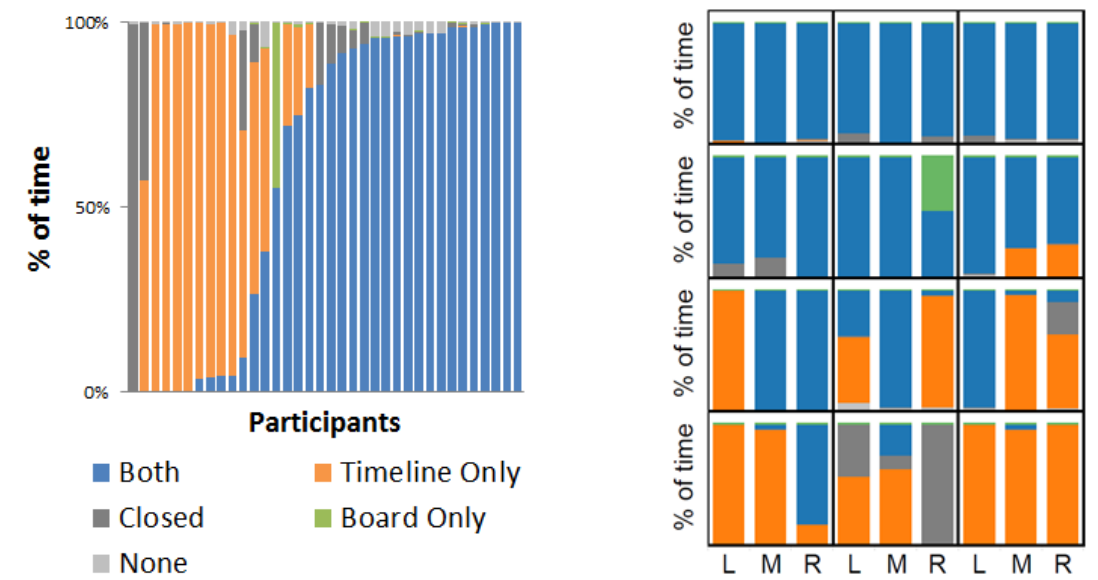

Fig. 4 Percent of time each timeline configuration was kept by each participant. (Left) Sorted by percent of time a participant kept the Both configuration. (Right) Sorted by the average percent of time a group kept the Both configuration. Each cell shows a group (12 groups in total) and each bar shows one participant, arranged by their seats (L: Left, M: Middle; R: right).

We further examined participants' usage of timeline configurations as groups, and found that most groups had at least one player keeping Both feedback mode on for most of the gameplay (see Fig. 4 Right). The last three groups (Group 2, 5, and 8 on the last row of Fig. 4 Right) all explicitly discussed the potential interference of displaying feedback on the map, while participants in Group 2 specifically agreed that only one player would be displaying feedback on the map.

\subsection{System Feature Usage for Maintaining Situation Awareness}

As the timeline was designed to improve users' situation awareness of dynamic changes, we examined the usage of the timelines in supporting the three levels of 
situation awareness (i.e., perception, comprehension, and projection) as defined by Endsley [11]. The first level of situation awareness, perception, refers to the knowledge of the changes that happened. In the context of the Pandemic game, the perception level refers to knowing what the dynamic changes were, as well as the whether the new changes were casual. The comprehension level refers to participants' understanding of the overall situation and of the changes that they just learned about to know their significance. Finally, the projection level refers to making predictions about future game states.

The three levels of situation awareness are internal cognitive processes. Thus, they are not directly observable without participants' verbal communication, physical interaction with the application interface, and visible body language. For example, participants may be exploring the timeline and thinking about the automated game events' impact on the overall game state. However, without verbal communication, it is impossible to definitely determine whether the interaction facilitated participants' comprehension. For this reason, few observable actions occurred for the comprehension level. Moreover, we incorporated decision making into the third level, projection, although it was originally modeled as a separate process by Endsley [11]. Participants' strategizing and prioritization behaviour represented participants' decisions in response to their projection of future game states. Since our data only recorded participants' visible and audible behaviours, we were constrained to determining how the timelines supported situation awareness based on observable actions.

We were also interested in how other system features were used for maintaining situation awareness. The video analysis revealed that the game map and the discard pile were the most relevant features used by participants. The game map refers to the connected cities as well as all information contained within it, e.g., the disease cubes on cities, locations of player pawns, and system animations that highlight particular cities. The discard pile contained a log of limited history of cities infected by automated events, periodically emptied after epidemic events. It could be opened by tapping a button, as described in section 4 above.

In the following sections, we present data pertinent to how the timelines, the game map, and the discard pile were used by our participants to gather situation awareness information for each level of situation awareness: perception, comprehension, and projection (as depicted in Fig. 5, Fig. 6, and Fig. 7).

\subsection{Perception}

At the end of each player turn, the system automated new game events, which included new player cards (i.e., shared resources) and new disease infections on the game map (i.e., changes in the system state). The new changes were reflected on the associated cities and were highlighted on the map by a brief system animation. Moreover, they were appended to the timelines (players had to tap on the new 
changes to see the associated locations). Participant should aim to find out about the details of all new events, including the type of events that took place, their locations and quantity, and if the events were causal to one another.

The analysis revealed that participants typically first observed both the system animation appeared on the game map and the timelines to gain awareness of dynamic changes. They often narrated the changes. However, participants sometimes only caught parts of complex changes or completely missed the changes. Timelines were then used to investigate new changes that they missed, and it was considered as the correct history account to facilitate discussions. In this section, we describe participants' behaviour for these two cases of automated changes, as depicted in Fig. 5.

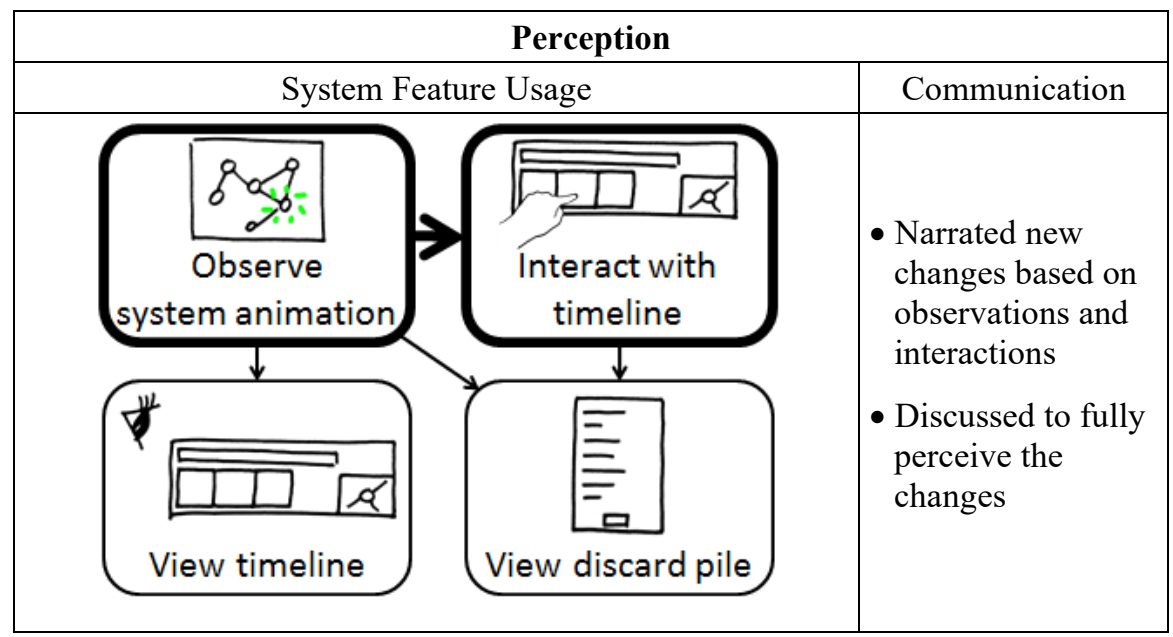

Fig. 5 At the perception level, participants typically first observed system animations and then interacted with their timelines to verify or further investigate changes. Changes were often narrated, and participants also discussed changes based on information gathered to negotiate their knowledge.

\subsubsection{Observation first then investigation}

Participants often narrated new changes as system animations appeared on the game map. Due to the large size of the tabletop display and the fact that players were not constantly attending to the interface during gameplay, players sometimes missed seeing the system animations in time or only noticed that some changes took place without knowing the details (e.g., they noticed an animation occurring in their peripheral view). Moreover, complex changes that involved chained events took time to animate while they could be difficult to follow. For example, after the system finished animating on the map, a player $\left(\mathrm{P}_{1, \text { middle }}\right)$ noticed it but did not know the exact associated cities from observing the map. He said to the 
group: "hmm... Something went pop!" He then used his timeline to locate the changes by tapping on the newly automated event and narrated the result to the group: "San Francisco." As he had Both feedback on, another player $\left(\mathrm{P}_{1, \text { left }}\right)$ then pointed at the replay animation triggered and said: "right here."

The timeline were also useful as a static visualization to perceiving new changes, especially because it automatically scrolled to show the current turn and placed the changes in a readily accessible location for users. There were only $9 / 333$ cases of such usage that we coded. However, the actual usage could be much higher due to the constraints in precisely determining the eye gazes of participants.

Although the static timeline design did not provide detailed location information of the game events, the colour-coding of game events provided a general sense of regions. The icons indicated the types of events (i.e., infection, outbreak, or epidemic), and the amount of game events provided a hint to the complexity of changes. Moreover, there were a few cases where participants opened and closed their timelines only to view the changes without interacting with any specific game events (evident from their narrations). Participants sometimes narrated the colour of the player cards (shared resources) received by collaborators, showing the value of providing awareness of the changes in shared resources automated by the system.

\subsubsection{Interactions to resolve complex events}

For complex disease spread, participants' process of learning the extent of the changes was often a joint, iterative effort among the team members. While the game map provided a reference to the current system state and allowed participants to notice changes, the timelines were the main tools for participants to understand how the system automation arrived at the new state.

Participants interacted with the timelines to verify what they observed on the game map or what they overheard from collaborators. The timeline was also used to investigate new changes. This was the most common type of timeline usage (272/333 cases). It was also common for multiple players to investigate on their timelines and announce the results at the same time. We hypothesize that players did so to make sure they, as a group, had the correct knowledge of the automation that took place.

When participants were confused with complex changes reflected on the game map, they used the timelines to investigate and verbalized their perception for negotiation to reach a common ground of the events that happened. In this process, the timeline was considered as the "correct" history and was used to correct each other's "theory" of the changes. For example, while the system animation was still playing, $\mathrm{P}_{3}$, right noticed that two outbreak events just took place by viewing his timeline, and he announced this to the group. As there were two outbreak events, participants tried to determine if one caused the other. It was a complex event as 
three types of events happened during the same turn: an epidemic event at Chennai, two independent outbreak events at Bangkok and New York, and an infection event at Moscow. As $\mathrm{P}_{3}$, right investigated using his timeline, the rest of the team looked at the game map on which they could see the new disease-spread system animation that was still playing, in addition to the replay animations triggered by him. After he identified that the first outbreak event occurred at Bangkok, by checking his timeline, $\mathrm{P}_{3}$, left mistakenly thought that it caused a chained outbreak event in Chennai. $\mathrm{P}_{3}$, middle then jumped in and tapped on the second game event with an outbreak icon, and this triggered a replay animation on the game map at New York. $\mathrm{P}_{3}$, right then continued to check game events on the timeline but provided an incorrect reasoning to why the two outbreaks were not chained. As $\mathrm{P}_{3}$, right had an incorrect reasoning, $\mathrm{P}_{3}$, left finally started interacting with the game events on his timeline and announced the correct set of events that took place: "It's with Bangkok and then New York. Those are the two outbreaks."

Our results showed that some participants appreciated the replay animation on the game map and commented that it was beneficial for keeping track of others' exploration on their timelines. However, some also noted that it distracted and confused them. Although the system animation and replay animation looked different and were colour-coded (see Fig. 3K and Fig. 3L for an example), participants confused them and had difficulties distinguishing these two types of animations quickly. For example, $\mathrm{P}_{5}$, left mistook the animation triggered by $\mathrm{P}_{5}$, middle as new outbreaks by the system, and announced "Bogota just outbroke!" He then quickly realized that it was a replay animation triggered by $\mathrm{P}_{5}$, middle, and said "oh no, you are just smashing things. I hate you! I hate the board thing! Turn your board off, please!" $\mathrm{P}_{5}$, middle, then turned off the feedback on the map. This confusion resulted in a negative response to the replay animation feature. Participants continued their discussion and pointed out that the key issue was the lack of awareness of collaborators' actions.

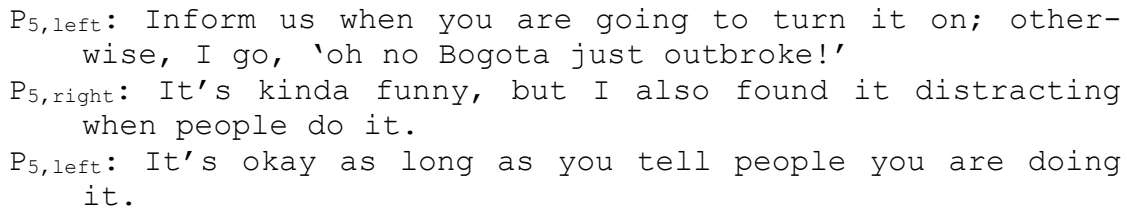

Due to the potential interference, some players manually toggled the feedback locations. However, this resulted in mode errors [39] where participants forgot about the current timeline mode and were confused when the replay animation was not triggered on the game map.

The discard pile system feature was used for perceiving new changes as well, although infrequently ( 8 cases vs. 281 cases for timelines). In 3 of these 8 cases, the discard pile was used in conjunction with the timelines to verify the changes. For example, after new changes took place during one group's gameplay, $\mathrm{P}_{9}$, middle was confused about why there was an additional disease cube on Moscow. He first navigated through the game history on his timeline to search for when it first hap- 
pened. $\mathrm{P}_{9}$, left then opened the discard pile to check. Then, $\mathrm{P}_{9}$, middle and $\mathrm{P}_{9}$, left found that Moscow happened in the most recent turn through the timeline and the discard pile, respectively.

Overall, players reached the correct perception of the automated events most of the time (293/397 cases, 68.26\%) even though participants had to correct themselves or each other in $22 / 293$ cases, $7.51 \%$. There were $99 / 397,24.94 \%$, cases in which we were unable to determine whether their perception was correct and $5 / 397$ cases, $1.26 \%$, where participants gained incorrect information or could not find the information needed.

The analysis also revealed the flexible work patterns employed by our participants. Participants sometimes ignored the system animations and continued to discuss strategy. Moreover, since advance planning of actions was common and necessary in the game, the current player sometimes focused on executing the actions agreed upon by the entire group beforehand, and relied on team members to observe and report the new changes. This finding showed the importance of providing persistent timelines for individuals to support such flexible work patterns.

\subsection{Comprehension}

With the new changes explored, the comprehension level refers to making sense of the new changes and the overall game state. The players should seek to determine how the new changes impacted the overall game state. As participants were all experienced Pandemic game players, they generally understood the meaning of the changes. In some cases, the new changes did not have urgent impact on the game state, while in other cases participants started strategizing about how to address changes right away. We based our analysis on observable behaviours, and our data showed that the game map was used as a reference point for the group to comprehend the overall game state.

The game map was the most frequently used in the comprehension level to understand the overall state as well as to spot inconsistency in their understanding of the game state, as depicted in Fig. 6. After new changes took place, participants commented on overall game state based on the game map. For example, in one session, $\mathrm{P}_{5}$, middle commented on the overall spread of the blue colour diseases on the game map: "Oh my goodness, there's a lot of blue going on!". 


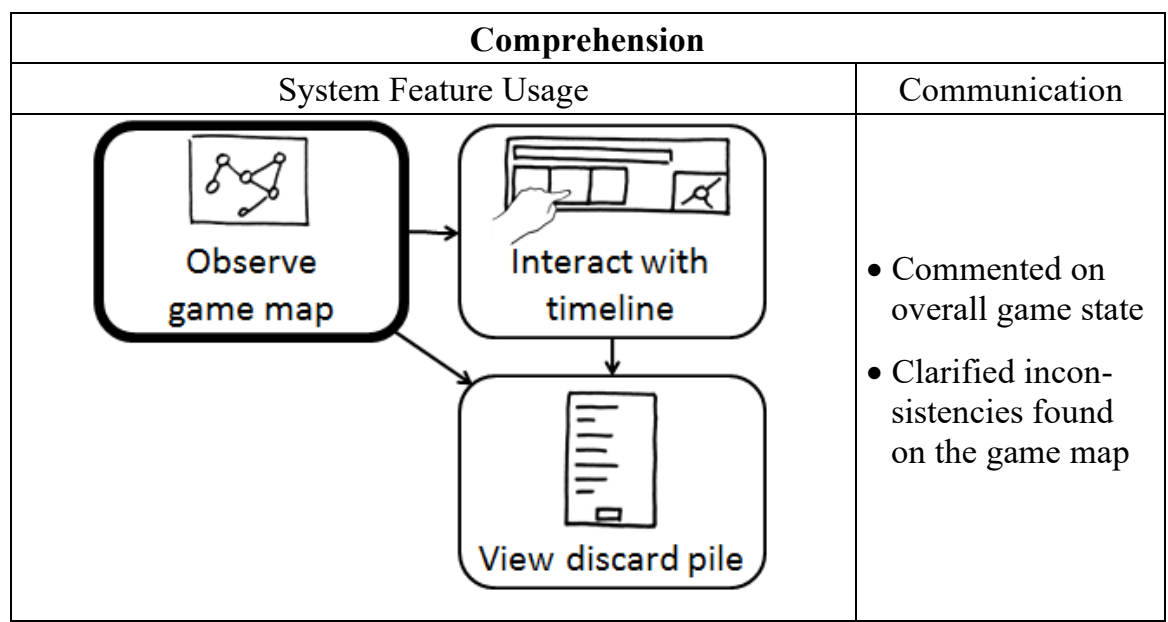

Fig. 6 At the comprehension level, the game map was used most often to discover inconsistencies between participants' understanding of the game state and the actual game state. The timeline and the discard piles were then used to gather information, which allowed them to collaboratively understand the game state.

The game map was also used in conjunction with the discard pile and timelines sometimes for players to correct their understanding of the system state. For example, $\mathrm{P}_{1 \text {,right }}$ noticed that Bogota had more disease cubes on it on the map than expected and asked "have we been noticing that Bogota is a problem?" Then, $\mathrm{P}_{1, \text { left }}$

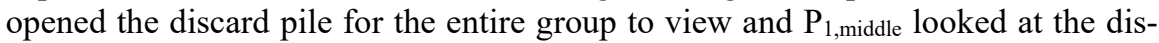
card pile and clarified that "no, it's just out [in the last turn]."

In another example, after new changes took place, $\mathrm{P}_{3 \text {,right }}$ first checked his timeline. Later on, while inspecting the game map, he found that the narrated events were inconsistent with the number of disease cubes on the map. This prompted $\mathrm{P}_{3 \text {,right }}$ to further interacted with his timeline to fully correct the group's knowledge, and he announced the correction to the group.

By the end of the comprehension stage, participants had usually reached agreement about the changes that took place and their meaning to the game. Next, they negotiated with teammates on what strategies to take and which actions to prioritize.

\subsection{Projection}

The projection level refers to predicting the future game state, and participants strategized, prioritized actions, and managed resources, based on their predictions. Generally in the game, players need to strategize based on when critical events would happen at which locations. This information can be estimated based on the 
current and past disease spread as well as when previous critical events took place. While the timeline and discard pile were both the key system features used to help remember historical events and forecast future game states, the discard pile was the primary feature used, as depicted in Fig. 7.

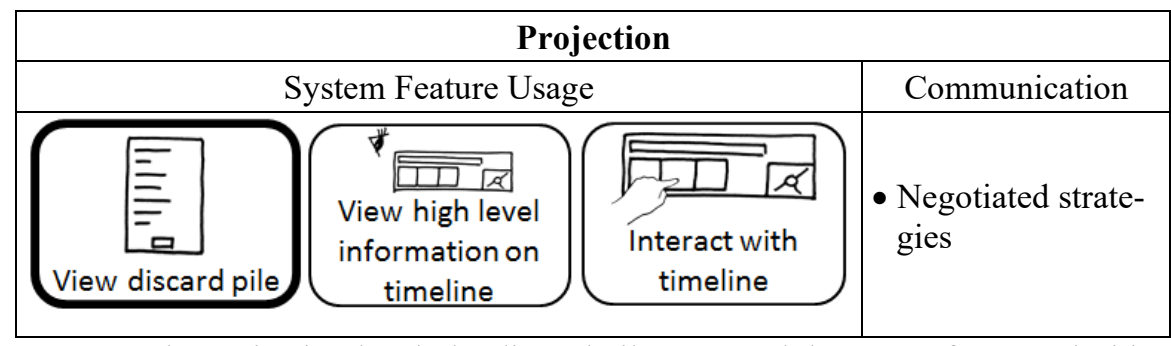

Fig. 7 At the projection level, the discard pile was used the most often to prioritize actions, and participants negotiated their strategies with each other based on the information gathered. The timeline was beneficial for participants to view high level information, but it was used less frequently.

We found that the timeline provided high-level information that was beneficial for forecasting game states. For example, players counted the number of turns since the last epidemic event on the overview bar at the top of the timelines $(6 / 333$ cases). As a fix number of epidemic events were roughly evenly distributed in the game, counting the number of turns since the last epidemic is a good predictor of the next epidemic event. Epidemic events signaled that previous infected cities may be infected again soon to create wider disease spread, so it was important for players to predict when the next epidemic event might occur and adapt their strategies accordingly. Players also navigated through historical events to determine if any cities might be potential problems in the future. Since all other system features only provided limited amounts of historical information (e.g., the discard pile only listed the infected cities since the last epidemic event), players had to rely on timelines for much older events.

The analysis showed that very few timeline interactions were conducted for the purpose of strategizing (used only 6 times in 88 cases of prioritization). The amount of effort required to navigate through many turns to locate the target game event likely contributed to the limited use of the timeline for this purpose. More often, participants opened and read the content of the discard pile to prioritize their actions (used 82 times). The discard pile provided a quick way to access recent cities that were affected by disease spreads by locating all information in a single textual log with minimal interaction required (other than to open, and potentially reposition the widget). This information allowed players to decide which cities needed more attention by comparing cities in the pile and the current game state on the map. This showed that the design of such a textual log was more beneficial for the projection level of situation awareness.

The following example illustrates a usage of the discard pile for the purpose of prioritizing actions. $\mathrm{P}_{1 \text {,middle }}$ went through a list of cities that could potentially cre- 
ate outbreaks based on the current game state (i.e., cities that needed more urgent attention). $\mathrm{P}_{1 \text {,middle }}$ first named Moscow and $\mathrm{P}_{1 \text {, left }}$ opened the discard pile for the entire group to see (default location is at the centre of the map). After confirming that it was not in the pile and thus was potentially high in priority, $\mathrm{P}_{1 \text {,middle }}$ continued to inquire the group about the status of Mumbai and Bangkok. $\mathrm{P}_{1 \text {,left }}$ opened the discard pile again, and $\mathrm{P}_{1 \text {,right }}$ viewed the pile and confirmed that they were in the pile, meaning that players only needed to attend to them when the next epidemic event is near. $\mathrm{P}_{1 \text {,middle }}$ thus concluded that Moscow was the only problematic city: "which is to say, Moscow is the only thing [to be concerned about]." $\mathrm{P}_{1 \text {,left }}$ agreed and reiterate on the urgency of Moscow: "that [Moscow] really needs to be dealt with right now." Players then continued to discuss how to spend actions to move to Moscow, and eventually treated diseases on Moscow in the same turn.

The discard pile was also sometimes used as a tool to suggest potential actions to consider. However, this sometimes failed because there was too much information to parse through (i.e., too many cards in the discard pile), or it was simply not helpful due to the game state at the time.

\section{Discussion}

While the data analysis in Phase 1 revealed no difference across timeline alternatives in group situation awareness, the follow up analysis showed that there was frequent communication among players to narrate information, discuss changes, and negotiate strategies, and this sharing of information facilitated the group situation awareness. The game map, timelines, and the discard piles acted as I information sources for situation awareness maintenance. In this section, we discuss specific timeline designs that were beneficial for participants' situation awareness and lessons learned for potential improvement.

\subsection{Perception: Make Changes Readily Available}

The timelines were mainly used for perceiving new automated changes, and several aspects of the timelines helped participants to gather this information. The timelines appended new changes and automatically scrolled to the current turn, making the most recent information readily available for exploration. The visual design of the timeline structured the game events based on their types into three rows (i.e., player action vs. system automations) to facilitate locating game events. The colour-coding and icons used provided overview information. Moreover, each timeline was placed at individual's personal area to provide quick access to new changes, visually and physically. In contrast, the discard pile was used less frequently for perceiving new changes, and this may be due to the fact that reaching 
out to open the discard pile took more physical effort or required more coordination to ask the player on the left (the position closest to the discard pile button) to open it. This may also be why the shared timelines were used less frequently and resulted in lower situation awareness in Phase 1 of the study.

In light of the benefits of timelines, potential redesign may consider how to further streamline the perception of new changes, such as reducing the interaction required by including more detailed information for the most recent events.

\subsection{Projection: Provide Critical Event Overview and Summary View}

While the overview of critical events on the timeline helped participants determine the overall strategy, the discard pile was used much more for forecasting events and prioritizing actions. The interactivity of the timeline was beneficial for reducing clutter. However, it required too much cognitive and physical effort for the participants to gain an overview of the historical events to project the relative urgency of problems. Moreover, the discard pile appeared by default at the centre of the game map, and this might have better facilitated information sharing and strategizing for a tightly coupled collaboration [43], such as in our context. Future designs of tabletop applications involving dynamic changes should consider providing a different summary view of recent events in the timeline or as a different system feature to support projection of future system states.

\subsection{Timelines for Supporting Group Work}

The timeline was designed to support situation awareness for collaborative work. Our analysis revealed that the timeline was often used in conjunction with the game map. While the game map reflected the current system state and helped participants to notice new changes, the timeline was used primarily as the correct historical account to negotiate users' perception of the changes.

We designed the replay animation, invoked by tapping game events, as a way for users to gain more detailed information of new changes and as a way to virtually point on the map for information sharing. While both use cases were found in the data, there were only a few clearly observable instances. Participants mostly physically pointed at the game map to aid their conversation, and we believe that this is due to the turn-based nature of the game and the difficulties in searching for the replay animation due to the current design and large size of tabletop displays.

Moreover, the replay animation sometimes confused the participants and they mistook the replay animations as new system automated events. Since having re- 
play feedback on the map and map cut-out on the timeline were the most popular configuration, future designs should consider more salient workspace awareness cues for the replay animation to facilitate feedthrough [29]. Considering participants' feedback in Phase 1 that the timeline felt disconnected from the game, we may consider a design where the timeline is visually associated with the replay animation to create a redundant encoding of invoker identity and allow for quicker association. Furthermore, as participants tried to manually toggle the feedback locations, they sometimes forgot about their current setting. A potential solution would be to use a user-maintained mode [39] for the replay animation, and replay animation is only displayed on the game map when users dwell on a game event on the timeline.

\subsection{Support Flexible Work Patterns}

Our data analysis revealed several work patterns. Although groups' collaboration styles were mostly tightly coupled, they often investigated their timelines concurrently to investigate changes and verify information observed from the game map or overheard from others. Moreover, they sometimes split the workload by having one participant carry out strategies previously agreed upon and having the rest of the team investigate changes. Phase 1 and Phase 2 data analysis revealed the benefits of individual timeline on improved situation awareness as well as the flexibility to allow participants to investigate changes at their own pace.

\section{Conclusion and Future Work}

Our video analysis showed the benefits of our interactive event timeline design for users to maintain situation awareness, especially for investigating automated system events. It was used as both static and interactive visualization. Our results showed that while the timeline was used to support the projection of future system states for the purpose of prioritizing actions, it can be further improved to provide summarized historical information.

In the future, we would like to investigate the following timeline redesign: streamlining the perception of new changes, providing a summary view to facilitate strategizing, and enhancing the replay animation to better facilitate observation of collaborators' actions. Furthermore, we would like to deploy the system in a home or game shop environment, which will have more interruptions in the environment. We would like to study the different needs that arise and understand the necessity of providing different types of information on the timelines. Finally, we would like to apply our findings to other domains, such as command and control and emergency response, as a debriefing tool for training purposes. Timelines 
can also be applied to other co-located environments, where a person in a monitoring role needs to keep track of the activities happening in the workspace. For example, two teachers co-teaching in a multi-device classroom also need a way to keep track of students' activities.

\section{Acknowledgments}

We would like to thank the Games Institute at the University of Waterloo, the Leif exchange program, NSERC, NSERC SurfNet, GRAND NCE, and SSHRC IMMERSe for funding this project. We also thank Matt Leacock and Z-Man Games for giving us permission to use the Pandemic board game.

\section{References}

1. Bortolaso, C., Oskamp, M., Graham, T.C.N., and Brown, D. OrMiS : A Tabletop Interface for Simulation-Based Training. ITS, (2013), 145-154.

2. Carvalho, P.V.R., dos Santos, I.L., and Vidal, M.C.R. Safety Implications of Cultural and Cognitive Issues in Nuclear Power Plant Operation. Applied Ergonomics 37, 2 (2006), 211 23.

3. Chang, Y.-L.B., Scott, S.D., and Hancock, M. Supporting situation awareness in collaborative tabletop systems with automation. ITS, (2014), 185-194.

4. Conversy, S., Gaspard-Boulinc, H., Chatty, S., Valès, S., Dupré, C., and Ollagnon, C. Supporting Air Traffic Control Collaboration with a TableTop System. CSCW, (2011), 425434.

5. Domova, V., Vartiainen, E., Azhar, S., and Ralph, M. An interactive Surface Solution to Support Collaborative Work Onboard Ships. ITS '13, (2013), 265-272.

6. Döweling, S., Tahiri, T., Sowinski, P., Schmidt, B., and Khalilbeigi, M. Support for collaborative situation analysis and planning in crisis management teams using interactive tabletops. ITS, (2013), 273-282.

7. Durlach, P.J. Change Blindness and Its Implications for Complex Monitoring and Control Systems Design and Operator Training. Human Computer Interaction 19, 4 (2009), 423-451.

8. Endsley, M.R. and Jones, W.M. A model of inter- and intrateam situation awareness: Implications for design, training and measurement. In M. McNeese, E. Salas and M. Endsley, eds., New trends in cooperative activities: Understanding system dynamics in complex environments. Human Factors and Ergonomics Society, Santa Monica, CA, 2001, 46-67.

9. Endsley, M.R. Situation awareness global assessment technique (SAGAT). IEEE National Aerospace and Electronics Conference, (1988), 789-795.

10. Endsley, M.R. A Survey of Situation Awareness Requirements in Air-to-Air Combat Fighters. The International Journal of Aviation Psychology 3, 2 (1993), 157-168.

11. Endsley, M.R. Toward a theory of situation awareness in dynamic systems. Human Factors 37, 1 (1995), 32-64

12. Endsley, M.R. Direct Measurement of Situation Awareness : Validity and Use of SAGAT. In M.R. Endsley and D.J. Garland, eds., Situation Awareness Analysis and Measurement. Lawrence Erlbaum, Mahwah, NJ, 2000, 131-157. 
13. Gorman, J.C., Cooke, N.J., and Winner, J.L. Measuring team situation awareness in decentralized command and control environments. Ergonomics 49, 12-13 (2006), 1312-25.

14. Gutwin, C., Greenberg, S., and Roseman, M. Workspace Awareness in Real-Time Distributed Groupware : Framework, Widgets, and Evaluation. Proc. of HCI on People and Computers XI, (1996), 281-298.

15. Gutwin, C. and Greenberg, S. Design for Individuals, Design for Groups: Tradeoffs Between Power and Workspace Awareness. CSCW, (1998), 207-216.

16. Gutwin, C. and Greenberg, S. A descriptive framework of workspace awareness for real-time groupware. Computer Supported Cooperative Work 11, (2002), 411-446.

17. Ha, V., Inkpen, K.M., Mandryk, R.L., and Whalen, T. Direct intentions: The effects of input devices on collaboration around a tabletop display. TABLETOP, (2006).

18. Hart, S.G., California, M.F., and Staveland, L.E. Development of NASA-TLX (Task Load Index): Results of Empirical and Theoretical Research. In P. Hancock and N. Meshkati, eds., Human Mental Workload. Amsterdam, North Holland, 1988, 139-183.

19. Heath, C. and Luff, P. Collaboration and Control: Crisis Management and Multimedia Technology in London Underground Line Control Rooms. Computer Supported Cooperative Work 1, 1-2 (1992), 69-94.

20. Isenberg, P. and Fisher, D. Collaborative Brushing and Linking for Co-located Visual Analytics of Document Collections. Computer Graphics Forum 28, 3 (2009), 1031-1038.

21.John, M., Smallman, H.S., and Manes, D.I. Recovery from interruptions to a dynamic monitoring task: the beguiling utility of instant replay. Human Factors and Ergonomics Society Annual Meeting 49, 3 (2005), 473-477.

22. Kaber, D.B. and Endsley, M.R. Out-of-the-loop performance problems and the use of intermediate levels of automation for improved control system functioning and safety. Process Safety Progress 16, 3 (1997), 126-131.

23. Kruger, R., Carpendale, S., Scott, S.D., and Tang, A. Fluid Integration of Rotation and Translation. CHI, (2005), 601-610.

24. Moore, R.J., Gathman, E.C.H., Ducheneaut, N., and Nickell, E. Coordinating Joint Activity in Avatar-Mediated Interaction. CHI, (2007), 21-30.

25. Morris, M.R., Paepcke, A., Winograd, T., and Stamberger, J. TeamTag: Exploring Centralized versus Replicated Controls for Co-located Tabletop Groupware. CHI, (2006), 1273-1282.

26. Nacenta, M. a., Pinelle, D., Stuckel, D., and Gutwin, C. The effects of interaction technique on coordination in tabletop groupware. Graphics Interface, ACM Press (2007), 191-198.

27. Parasuraman, R., Sheridan, T.B., and Wickens, C.D. A Model for Types and Levels of Human Interaction with Automation. IEEE Transactions on Systems Man and Cybernetics Part A: Systems and Humans 30, 3 (2000), 286-297.

28. Parush, A., Kramer, C., Foster-Hunt, T., Momtahan, K., Hunter, A., and Sohmer, B. Communication and team situation awareness in the OR: Implications for augmentative information display. Journal of biomedical informatics 44, 3 (2011), 477-85.

29. Pinelle, D., Gutwin, C., and Greenberg, S. Task analysis for groupware usability evaluation : modeling shared-workspace tasks with the mechanics of collaboration. ToCHI 10, 4 (2003), 281-311

30. Rensink, R.A. Change Blindness. In McGraw-Hill Yearbook of Science \& Technology. McGraw-Hill, New York, 2005, 44-46.

31. Rittenbruch, M. and McEwan, G. An historical reflection of awareness in collaboration. In P. Markopoulos, B. De Ruyter and W. Mackay, eds., Awareness Systems. Springer London, London, 2009, 3-48.

32. Ryan, R.M., Rigby, C.S., and Przybylski, A. The Motivational Pull of Video Games: A SelfDetermination Theory Approach. Motivation and Emotion 30, 4 (2006), 344-360.

33. Salas, E., Dickinson, T.L., Converse, S.A., and Tannenbaum, S.I. Toward an understanding of team performance and training. In R.W. Swezey and E. Salas, eds., Teams: Their training and performance. Ablex Publishing, Westport, CT, US, 1992, 3-29. 
34. Salas, E., Prince, C., Baker, D.P., and Shrestha, L. Situation awareness in team performance: implications for measurement and training. Human Factors 37, 1 (1995), 123-136.

35. Sasangohar, F., Scott, S.D., and Cummings, M. 1. Supervisory-level interruption recovery in time-critical control tasks. Applied ergonomics 45, 4 (2014), 1148-1156.

36. Schmidt, K. The problem with 'awareness.' CSCW, (2002), 285-298.

37. Scott, S.D. and Carpendale, S. Theory of Tabletop Territoriality. In C. Müller-Tomfelde, ed., Tabletops - Horizontal Interactive Displays. Springer-Verlag, London, 2010, 357 - 385.

38. Scott, S.D., Mercier, S., Cummings, M.L., and Wang, E. Assisting Interruption Recovery in Supervisory Control of Multiple UAVs. Human Factors and Ergonomics Society Annual Meeting 50, 5 (2006), 699-703.

39. Sellen, A., Kurtenbach, G., and Buxton, W. The Prevention of Mode Errors Through Sensory Feedback. Human Computer Interaction 7, 2 (1992), 141-164.

40. Smallman, H.S. and John, M. St. CHEX (Change History Explicit): New HCI Concepts for Change AwarenessS. Human Factors and Ergonomics Society Annual Meeting, (2003), 528 532 .

41. Smith, K. and Hancock, P.A. Situation Awareness is adaptive, externally directed consciousness. Human Factors 37, 1 (1995), 137-148.

42. Spindler, M., Tominski, C., Schumann, H., and Dachselt, R. Tangible Views for Information Visualization. ITS, (2010), 157-166.

43. Tang, A., Tory, M., Po, B., Neumann, P., and Carpendale, S. Collaborative coupling over tabletop displays. CHI, (2006), 1181-1190.

44. Tuddenham, P. and Robinson, P. Territorial Coordination and Workspace Awareness in Remote Tabletop Collaboration. CHI, (2009), 2139-2148.

45. Tuddenham, P. and Robinson, P. Coordination and awareness in remote tabletop collaboration. In C. Müller-Tomfelde, ed., Tabletops - Horizontal Interactive Displays. Springer-Verlag, London, 2010, 407-434.

46. Voelker, S., Weiss, M., Wacharamanotham, C., and Borchers, J. Dynamic Portals: A Lightweight Metaphor for Fast Object Transfer on Interactive Surfaces. ITS, (2011), 158 161 .

47. Wallace, J.R., Pape, J., Chang, Y.-L.B., et al. Exploring Automation in Digital Tabletop Board Games. Proc. of Computer Supported Cooperative Work Companion, (2012), 231234.

48. Wellens, A.R. Group situation awareness and distributed decision making: From military to civilian applications. In J. Castellan, ed., Individual and group decision making: Current Issues. Lawrence Erlbaum, Hillsdale, NJ, 1993, 267-291.

49. Yoshikawa, T., Shizuki, B., and Tanaka, J. HandyWidgets : Local Widgets Pulled-out from Hands. ITS, (2012), 197-200. 